\title{
Screening via CT angiogram after traumatic cervical spine fractures: narrowing imaging to improve cost effectiveness. Experience of a Level I trauma center
}

\author{
Megan M. Lockwood, MD, ${ }^{1}$ Gabriel A. Smith, MD, ${ }^{1}$ Joseph Tanenbaum, BS, ${ }^{1}$ Daniel Lubelski, MD, ${ }^{2}$ \\ Andreea Seicean, MD, PhD, MPH, Jonathan Pace, MD, ${ }^{1}$ Edward C. Benzel, MD, ${ }^{3}$ \\ Thomas E. Mroz, MD, ${ }^{3}$ and Michael P. Steinmetz, MD ${ }^{3}$
}

${ }^{1}$ Department of Neurological Surgery, Case Western Reserve University School of Medicine, MetroHealth Medical Center; ${ }^{2}$ Cleveland Clinic Lerner College of Medicine; and ${ }^{3}$ Cleveland Clinic Center for Spine Health, Cleveland, Ohio

\begin{abstract}
OBJECT Screening for vertebral artery injury (VAI) following cervical spine fractures is routinely performed across trauma centers in North America. From 2002 to 2007, the total number of neck CT angiography (CTA) studies performed in the Medicare population after trauma increased from 9796 to 115,021. In the era of cost-effective medical care, the authors aimed to evaluate the utility of CTA screening in detecting VAl and reduce chances of posterior circulation strokes after traumatic cervical spine fractures.
\end{abstract}

METHODS A retrospective review of all patients presenting with cervical spine fractures to Northeast Ohio's Level I trauma institution from 2002 to 2012 was performed.

RESULTS There was a total of 1717 cervical spine fractures in patients presenting to Northeast Ohio's Level I trauma institution between 2002 and 2012. CTA screening was performed in 732 patients, and 51 patients (0.7\%) were found to have a VAl. Fracture patterns with increased odds of VAI were C-1 and C-2 combined fractures, transverse foramen fractures, and subluxation of adjacent vertebral levels. Ten posterior circulation strokes were identified in this patient population $(0.6 \%)$ and found in only 4 of 51 cases of VAI (7.8\%). High-risk fractures defined by Denver Criteria, VAl, and antiplatelet treatment of VAI were not independent predictors of stroke.

CONCLUSIONS Cost-effective screening must be reevaluated in the setting of blunt cervical spine fractures on a caseby-case basis. Further prospective studies must be performed to elucidate the utility of screening for VAl and posterior circulation stroke prevention, if identified.

http://thejns.org/doi/abs/10.3171/2015.6.SPINE15140

KEY WORDS cervical spine fracture; vertebral artery injury; CT angiography; trauma

$\mathrm{S}$ CREENING for blunt cerebrovascular injuries (BCVI) in the setting of cervical spine fractures has been institutionalized at many Level I trauma centers across the United States. The incidence of vertebral artery injuries (VAIs) is estimated at $0.7 \%$ of all patients with blunt traumatic injuries. ${ }^{8,9,21,36}$ Without prompt treatment, devastating consequences, such as posterior circulation strokes, may occur. This risk has led many authors to advocate for strict screening of this patient population. ${ }^{4,16}$ There is no high-level evidence nor guidelines for effective screening for BCVI, $24,34,35$ but emerging data recommending aggressive screening for BCVI to prevent stroke have led many trauma centers to establish protocols for the use of multidetector CT angiography (CTA) to evaluate for BCVI in the emergency department. ${ }^{6,13-15,18}$
The cost effectiveness of screening for VAI continues to be a point of debate. Some centers have limited screening measures to only those patients identified as being at highrisk for VAI as defined by the Denver Criteria. ${ }^{4-7}$ "High risk" includes upper cervical spine fractures involving C-1, $\mathrm{C}-2, \mathrm{C}-3$, fractures through the transverse foramen, and subluxation at the facet joints. However, even in the highrisk patient population, some have questioned the utility of CTA, as surgical treatment strategies are often made independent of such information and treatment for VAI may be contraindicated due to other organ injuries. ${ }^{6,29,31,33}$ Although the complications of VAI may be severe, the incidence of posterior circulation strokes are even rarer, and functional outcomes after VAI are good..$^{1,4,16,20,33}$ In the present study, the authors evaluated CTA screening in traumatic cervical

ABBREVIATIONS BCVI = blunt cerebrovascular injury; $C T A=C T$ angiography; $V A I=$ vertebral artery injury .

SUBMITTED February 1, 2015. ACCEPTED June 17, 2015.

INCLUDE WHEN CITING Published online November 27, 2015; DOI: 10.3171/2015.6.SPINE15140. 
spine fractures over a 10 -year period in an attempt to elucidate subsets of patients for whom CTA imaging should be performed, and to better characterize the risk stratification of stroke from VAI found on CTA imaging.

\section{Methods}

A retrospective review of all adult patients with cervical spine fractures presenting to MetroHealth Medical Center, Cleveland, Ohio, from January 2002 to December 2012 was performed. The MetroHealth institutional review board committee approved the study protocol (IRB13-00712), and patient data were accessed through the Northern Ohio Trauma System prospective data registry. For each patient, radiology reports were reviewed for fracture type and level. Patient sex, age, and mortality were also recorded. Each CTA report was reviewed to identify VAI and, if present, the subsequent treatment of VAI. International Classification of Diseases, Revision 9, Codes 433-438 ("occlusion and stenosis of precerebral arteries," "occlusion of cerebral arteries," "transient cerebral ischemia," "acute but ill-defined cerebrovascular disease," "other and ill-defined cerebrovascular disease," and "late effects of cerebrovascular disease") were used to identify all patients with stroke within 30 days of injury. Follow-up was reviewed on all strokes for 90 days. Patients' strokes were excluded if the etiology was hemorrhagic or in the anterior circulation. All strokes included were confirmed on MRI or CT scans. Patients were excluded if they were younger than 18 years old, had chronic fractures, received MR angiography imaging rather than a CTA, or if there was an indeterminate radiology interpretation of whether a fracture was present.

\section{Screening}

Screening for blunt VAI was done using a modification of the Denver Criteria. High-risk patients were defined as those with cervical spine fractures of the vertebral body, pedicle, or transverse foramen; basilar skull fractures involving the carotid canal or petrous bone; Lefort II and III fractures; and penetrating injuries. Denver Criteria Grade 1 injuries were defined as a vessel lumen stenosis of less than $25 \%$, Grade 2 injuries as stenosis of the vessel lumen between $25 \%$ and $50 \%$, Grade 3 injuries as stenosis of the vessel greater than $50 \%$ or the development of a pseudoaneurysm, Grade 4 injuries as complete vessel occlusion, and Grade 5 injuries as complete transection of the artery. A fellowship-trained neuroradiologist made all initial reports of the presence and grade of cervical vascular injury. A staff neurosurgeon then confirmed the injury and decided on the desired follow-up and treatment. Treatment was individualized for each patient based on the extent of injury and presence of other injuries.

\section{Statistical Analysis}

Pearson's chi-square test and ANOVA were used to compare categorical and continuous variables, respectively. A p value of less than 0.05 was considered statistically significant. Logistic regression univariate analysis (reported as odds ratio and $95 \% \mathrm{CI}$ ) was used to assess likelihood between patient characteristics and VAI and stroke.

\section{Results}

From January 2002 to December 2012, 1717 patients presented with cervical spine fractures to our trauma center. After exclusion screening, 1435 patients remained in our study (Fig. 1). Of the 1435 patients, 732 underwent CTA for VAI screening, whereas 703 did not. The patients' mean age was 55 years and $66 \%$ of the population was male. Fractures at $\mathrm{C}-1$ represented $13 \%$ of the total, $20 \%$ occurred at C-2, and $1.1 \%$ were combined C-1 and C-2 fractures (Table 1). Fractures at C-3 accounted for $6 \%$, and the remaining nearly $65 \%$ of fractures occurred between C-4 and C-7 (Table 1). Based upon application of the Denver Criteria for high-risk cervical spine fractures associated with VAI, there were 871 high-risk fractures and 564 low-risk fractures (Fig. 1). CTA screening was obtained in 542 high-risk and 190 low-risk fractures, whereas 329 high-risk and 374 low-risk fractures were not screened. Fracture patterns were evaluated to assess for trends in CTA use (Table 1). Fractures of C-1, C-2, combined C-1 and $\mathrm{C}-2, \mathrm{C}-3$, and those involving the transverse foramen were statistically significant predictors of CTA screening, whereas subluxation or subaxial cervical spine fractures were not. Thus, the odds of receiving a CTA screening for a high-risk fracture were statistically significant (OR 3.2; 95\% CI 2.6-4.0).

Among patients receiving CTA, $7 \%(n=51)$ were found to have VAI, including 50 high-risk and 1 low-risk fractures (Table 2). Twenty-five patients had VAI Grades 1 and 2, and 26 patients had VAI Grade 3, 4, or 5. Associated carotid artery injury was found in 7 of 51 patients. Univariate analysis showed that combined $\mathrm{C} 1-2$ fractures, subluxation of adjacent vertebral levels, and transverse foramen fractures were independent predictors of VAI (Table 3). Of all patients with cervical spine fractures in the study, there were 10 who had posterior circulation strokes within 30 days from presentation, but only 4 were in the setting of a VAI (Table 4). Of the 4 patients with strokes due to VAI, 2 were discharged neurologically intact and 2 died secondary to polytrauma injuries. Of the 6 patients who had strokes without VAI, 3 had atrial fibrillation and embolic complications, and the etiology of 3 were likely multifactorial, with 1 stroke occurring after hospital admission. On CT imaging, there were 3 cerebellar infarcts and 3 posterior cerebral artery territory infarcts. Univariate analysis revealed an increased chance of developing a posterior circulation stroke after a cervical spine fracture was independent of the presence of VAI identified during screening (OR 2.3; 95\% CI 0.6-8.7), and high-risk fracture patterns did not increase the risk for a posterior circulation stroke (OR 1.5; 95\% CI 0.4-5.9). However, fracture patterns with reported subluxation (OR 6.7; 95\% CI 1.3-35.4) and transverse foramen involvement (OR 5.0; 95\% CI 1.1-22.7) were found to have an increased likelihood of a posterior circulation stroke in patients screened with CTA (Table 5).

Treatment for the 51 patients with VAI was heterogeneous in 90 days of follow-up. Thirty-five patients received antiplatelet medication and 5 were initially treated with anticoagulation before transitioning to aspirin by 3 months. Eleven patients were not treated with bloodthinning medication, and 1 patient died of other injuries 
1717 pts presented to ED with C spine fracture (1/02-12/12)

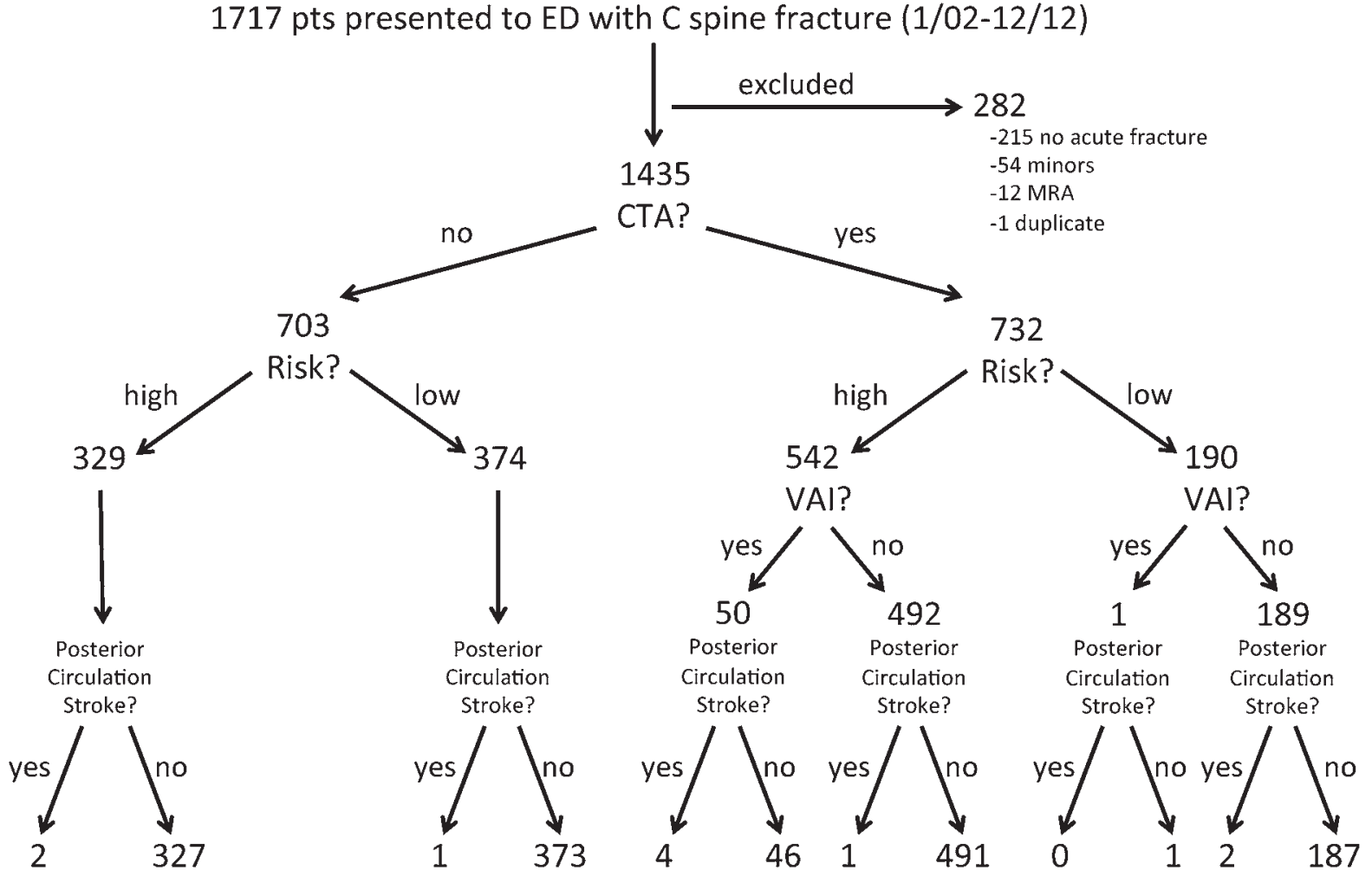

High-risk fractures: 3.2 OR getting CTA vs low-risk

Received CTA: 2.3 OR having stroke 1.5 OR having stroke vs low-risk

FIG. 1. Flow chart of CTA screening in 1717 patients presenting to MetroHealth Medical Center with traumatic cervical spine fractures. $\mathrm{C}$ spine = cervical spine; $\mathrm{ED}=$ emergency department; $M R A=M R$ angiogram; pts = patients.

TABLE 1. Patient characteristics according to CTA screening

\begin{tabular}{|c|c|c|c|c|}
\hline \multirow[b]{2}{*}{ Characteristic } & \multicolumn{2}{|c|}{ CTA } & \multirow[b]{2}{*}{$p$ Value* } & \multirow[b]{2}{*}{$\%$ of Total Population } \\
\hline & No & Yes & & \\
\hline No. of patients (\%) & $703(49.0 \%)$ & $732(51.0 \%)$ & & 1435 \\
\hline Male sex, $\%$ & 66.2 & 59.2 & $<0.01$ & 62.6 \\
\hline Mean age (median), yrs \pm SD & $54 \pm 22(52)$ & $55 \pm 22(54)$ & 0.37 & $55 \pm 22(53)$ \\
\hline \multicolumn{5}{|l|}{ Type of cervical spine fracture, $\%$} \\
\hline C-1 & 13.1 & 17.8 & 0.01 & 15.5 \\
\hline $\mathrm{C}-2$ & 20.3 & 33.9 & $<0.001$ & 27.3 \\
\hline Combined C-1 \& C-2 & 1.1 & 7.8 & $<0.001$ & 4.5 \\
\hline $\mathrm{C}-3$ & 5.7 & 8.3 & 0.05 & 7.0 \\
\hline Combined C4-7 & 64.4 & 44.5 & $<0.001$ & 54.3 \\
\hline Subluxation & 4.8 & 5.9 & 0.38 & 5.4 \\
\hline Transverse foramen fracture & 7.4 & 21.3 & $<0.001$ & 14.5 \\
\hline High-risk cervical spine fracture per Denver Criteria & 46.8 & 74.0 & $<0.001$ & 60.7 \\
\hline VAI, $\%$ & NA† & 7.0 & NA† & 3.6 \\
\hline Stroke, \% & 0.4 & 1.0 & 0.23 & 0.7 \\
\hline Mortality, \% & 1.3 & 1.4 & 0.89 & 1.3 \\
\hline
\end{tabular}


TABLE 2. Summary of cervical spine fractures, arterial injury, and treatment characteristics

\begin{tabular}{|c|c|}
\hline Characteristic & No. of Patients \\
\hline \multicolumn{2}{|l|}{ Type of cervical spine fracture } \\
\hline C-1 & 6 \\
\hline $\mathrm{C}-2$ & 12 \\
\hline Combined C-1 \& C-2 & 12 \\
\hline Subaxial fractures & 21 \\
\hline Subluxation & 10 \\
\hline Transverse foramen fracture & 32 \\
\hline \multicolumn{2}{|l|}{ VAl grade } \\
\hline 1 or 2 & 25 \\
\hline 3 or 4 & 26 \\
\hline \multicolumn{2}{|l|}{ Associated CAI } \\
\hline Yes & 7 \\
\hline No & 44 \\
\hline \multicolumn{2}{|l|}{ Treatment } \\
\hline Antiplatelet & 35 \\
\hline Anticoagulant & 6 \\
\hline Endovascular & $1^{*}$ \\
\hline Contraindicated & 11 \\
\hline Deceased & 1 \\
\hline \multicolumn{2}{|l|}{ Follow-up imaging } \\
\hline None & 23 \\
\hline DSA & 5 \\
\hline CTA & 19 \\
\hline MRA & 6 \\
\hline
\end{tabular}

$\mathrm{CAI}=$ carotid artery injury; DSA = digital subtraction angiography.

* Only 1 for VAl and 1 for carotid artery injury.

prior to initiating treatment. Of the 11 patients receiving no treatment, 1 developed a stroke (11\%); of the 35 patients receiving antiplatelet therapy, 2 developed a stroke (5.7\%). One patient underwent coil embolization after VAI because a pseudoaneurysm was of concern on CTA. Of the 5 patients who received anticoagulation therapy, 1 developed a stroke (20\%). The different treatment modalities

TABLE 3. Factors associated with 51 VAls in 732 patients who underwent CTA screening

\begin{tabular}{lc}
\hline \multicolumn{1}{c}{ Factor } & OR $(95 \% \text { Cl })^{*}$ \\
\hline Male sex & $1.6(0.8-2.9)$ \\
\hline Age, per 5 yrs & $1.0(0.9-1.0)$ \\
\hline Type of cervical spine fracture & \\
\hline C-1 & $1.1(0.6-2.3)$ \\
\hline C-2 & $1.1(0.6-1.9)$ \\
\hline Combined C-1 \& C-2 & $3.8(1.8-7.9)$ \\
\hline C-3 & $2.2(1.0-4.9)$ \\
\hline Combined C4-C7 & $1.0(0.6-1.8)$ \\
\hline Subluxation & $4.8(2.2-10.4)$ \\
\hline Transverse foramen fracture & $6.3(3.5-11.4)$ \\
\hline High-risk cervical fracture per Denver Criteria & $19.2(2.6-139.9)$ \\
\hline
\end{tabular}

* Boldfaced values are statistically significant.
TABLE 4. Characteristics of 10 patients with cervical spine fractures who had posterior circulation stroke

\begin{tabular}{lc}
\hline \multicolumn{1}{c}{ Characteristic } & No. of Patients \\
\hline C-1 & 1 \\
\hline C-2 & 3 \\
\hline Combined C-1 \& C-2 & 1 \\
\hline Subaxial fractures & 5 \\
\hline Subluxation & 2 \\
\hline Transverse foramen fracture & 4 \\
\hline VAl grade by CTA & \\
\hline 1 or 2 & 0 \\
\hline 3,4, or 5 & 4 \\
\hline No VAl & 4 \\
\hline No CTA & $2^{*}$ \\
\hline Associated CAI by CTA & 2 \\
\hline Yes
\end{tabular}

* Both patients had strokes unrelated to cervical spine fracture.

did not correlate with an increased incidence of stroke ( $\mathrm{p}$ $=0.8$.

\section{Discussion}

Aggressive screening protocols for VAI in the setting of acute traumatic cervical spine fracture have rapidly increased in the past 2 decades. ${ }^{23,26-28,30,32}$ Originally thought to be benign, VAI has been shown to confer a small but significant stroke risk. ${ }^{4,5,22}$ Trauma centers throughout the United States have begun implementing protocols to screen for cerebrovascular injury using CTA among those patients with penetrating injuries, LeFort II/III fractures, high-risk Denver Criteria cervical spine fractures, temporal bone fractures involving the carotid canal, and for unexplained ischemic events. ${ }^{2,3,5,6,8,10,13}$ Catheter-based diagnostic cerebral angiography remains the gold standard but it is an invasive procedure with significant cost and required resources. Its many known risks include complica-

TABLE 5. Preoperative factors associated with stroke in 732 patients who underwent CTA screening

\begin{tabular}{lc}
\hline \multicolumn{1}{c}{ Factor } & OR $(95 \% \text { Cl })^{*}$ \\
\hline Male sex & $0.9(0.2-4.1)$ \\
\hline Age, per 5 yrs & $0.9(0.8-1.1)$ \\
\hline Type of cervical fracture & \\
\hline C-1 & $0.8(0.1-6.5)$ \\
\hline C-2 & $0.3(0.0-2.7)$ \\
\hline Combined C-1 \& C-2 & $2.0(0.2-16.8)$ \\
\hline C-3 & NA† \\
\hline Combined C4-7 & $1.7(0.4-7.5)$ \\
\hline Subluxation & $6.7(1.3-35.4)$ \\
\hline Transverse foramen fracture & $\mathbf{5 . 0}(1.1-22.7)$ \\
\hline High-risk cervical fracture per Denver Criteria & $0.9(0.2-4.5)$ \\
\hline * Boldfaced values are statistically significant. \\
$\begin{array}{l}\dagger \text { Too few patients with C-3 fractures had strokes to be able to determine } \\
\text { the OR. }\end{array}$
\end{tabular}


tions related to catheter insertion, contrast administration, and stroke. ${ }^{6,32}$ In addition, time constraints to obtain the study make CTA more cost-effective if screening is going to be performed..$^{11,13}$

In this study, we evaluated the utility of CTA for screening VAI and the incidence of VAI after traumatic cervical spine fractures. In 1435 patients with cervical spine fractures, only those involving C-1, C-2, C-3, combined $\mathrm{C}-1$ and $\mathrm{C}-2$, and transverse foramen fractures were predictors of CTA screening at our institution-all of which are high-risk fracture patterns per the Denver Criteria. ${ }^{6}, 12,19,26,35$ Among screened patients, only combined $\mathrm{C}-1$ and $\mathrm{C}-2$ fractures, subluxation, and transverse foramen involvement were predictive of VAI. Specifically, the odds of developing a VAI were 3.8 (95\% CI 1.8-7.9), 4.8 (95\% CI 2.2-10.4), and 6.3 (95\% CI 3.5-11.4) for these fracture patterns, respectively. Of the 1435 patients with cervical spine fractures, only $10(0.7 \%)$ suffered a posterior circulation stroke, and of the 732 undergoing CTA, only 4 patients $(0.5 \%)$ had strokes in the presence of VAI.

Thus, the clinical significance of screening remains in doubt. Other institutions have reported a low incidence of posterior circulation strokes and VAI, as well. Scott et al. reviewed 187 patients who presented with Grade 1 and 2 VAI after blunt trauma. ${ }^{36}$ Two-thirds of patients were treated with aspirin, whereas one-third were unable to receive medication secondary to other injuries. They noted posttraumatic cerebral infarction rates of in 2 of the 187 patients (1.7\%), and both patients were taking aspirin. The authors found no correlation between treatment of VAI and prevention of a posterior circulation stroke. In another case series, Eastman et al. examined 26 patients with either blunt carotid or blunt VAI and found only 1 patient (3.9\%) with an infarct, which occurred from a Grade 3 injury. ${ }^{17,18}$ In our study, of the 51 patients suffering from VAI, 11 (20\%) received no treatment, $35(68.6 \%)$ received antiplatelet therapy, and 5 (11.8\%) received anticoagulation therapy, and we also found no correlation with treatment and prevention of stroke.

Our data suggest that screening for isolated VAI after cervical spine fractures should be reexamined. We did not find a significant association between Denver Criteria high-risk cervical spine fractures and posterior circulation strokes. We did, however, isolate 3 patterns-combined C-1 and C-2 fractures, subluxation of adjacent vertebral levels, and fractures through the transverse foramenthat were independently predictive of VAI. Furthermore, subluxation and transverse foramen fractures did have an increased likelihood of subsequent posterior circulation strokes, and screening should be strongly considered when these fracture types are identified. These data highlight the need for further studies and Level I evidence to evaluate this screening process. Additional validation from prospective studies is needed.

Neck CTA costs approximately $\$ 708$ to perform, with published values ranging from $\$ 500$ to $\$ 1500$ at various institutions. ${ }^{13,25}$ From 2002 to 2007 , the total number of neck CTAs performed in the Medicare population nationally in the United States increased from 9796 to 115,021, which would translate into a cost increase from $\$ 6.94$ million to $\$ 81.4$ million. These estimates do not take into ac- count subsequent follow-up imaging, radiology interpretation, subspecialty clinic visits, blood work, or complications related to treatment of asymptomatic VAI. Thus, a more specific screening tool must be developed to target those at high-risk for severe complications after VAI.

Although a high-risk fracture pattern was a statistically significant predictor of undergoing screening, 329 high-risk patients did not undergo screening. This can be explained by outside hospital transfer patients who may have already had screening, or by fracture patterns that, upon clinical interpretation by a spine specialist, were not thought to be at high risk for VAI. Our study has several other limitations. This was a retrospective analysis and, therefore, has the associated biases and limitations. While the initial screening pool was large and represented a 10year period, the sample size was low. As there were only 4 recorded stroke events among those with VAI, it is difficult to find a statistically significant difference comparing so few people. These data also represent a single-institution experience, which limits its broader application. Furthermore, while we can track the care a patient has received at our own hospital, we do not know if they sought care at another institution. Thus, the stroke risk may be underestimated.

\section{Conclusions}

CTA screening is an important tool to identify blunt cerebrovascular injury following trauma but should be used with caution in broad screening protocols for cervical spine fractures. Patients with C-1 and C-2 combined fractures, transverse foramen fractures, and subluxation of adjacent vertebral levels should be considered for CTA screening if clinical suspicion is present. These data support the need for Level I evidence to evaluate the cost effectiveness and efficacy of screening for and treating VAI in the setting of blunt trauma.

\section{References}

1. Alterman DM, Heidel RE, Daley BJ, Grandas OH, Stevens SL, Goldman MH, et al: Contemporary outcomes of vertebral artery injury. J Vasc Surg 57:741-746, 2013

2. Bagley LJ: Imaging of spinal trauma. Radiol Clin North Am 44:1-12, vii, 2006

3. Berne JD, Cook A, Rowe SA, Norwood SH: A multivariate logistic regression analysis of risk factors for blunt cerebrovascular injury. J Vasc Surg 51:57-64, 2010

4. Biffl WL, Moore EE, Elliott JP, Ray C, Offner PJ, Franciose RJ, et al: The devastating potential of blunt vertebral arterial injuries. Ann Surg 231:672-681, 2000

5. Biffl WL, Moore EE, Offner PJ, Brega KE, Franciose RJ, Burch JM: Blunt carotid arterial injuries: implications of a new grading scale. J Trauma 47:845-853, 1999

6. Biffl WL, Moore EE, Offner PJ, Brega KE, Franciose RJ, Elliott JP, et al: Optimizing screening for blunt cerebrovascular injuries. Am J Surg 178:517-522, 1999

7. Biffl WL, Moore EE, Offner PJ, Burch JM: Blunt carotid and vertebral arterial injuries. World J Surg 25:1036-1043, 2001

8. Bromberg WJ, Collier BC, Diebel LN, Dwyer KM, Holevar MR, Jacobs DG, et al: Blunt cerebrovascular injury practice management guidelines: the Eastern Association for the Surgery of Trauma. J Trauma 68:471-477, 2010

9. Burlew CC, Biffl WL: Blunt cerebrovascular trauma. Curr Opin Crit Care 16:587-595, 2010 
10. Burlew CC, Biffl WL, Moore EE, Barnett CC, Johnson JL, Bensard DD: Blunt cerebrovascular injuries: redefining screening criteria in the era of noninvasive diagnosis. $\mathbf{J}$ Trauma Acute Care Surg 72:330-337, 539, 2012

11. Burlew CC, Biffl WL, Moore EE, Pieracci FM, Beauchamp $\mathrm{KM}$, Stovall R, et al: Endovascular stenting is rarely necessary for the management of blunt cerebrovascular injuries. J Am Coll Surg 218:1012-1017, 2014

12. Cothren CC, Moore EE, Biffl WL, Ciesla DJ, Ray CE Jr, Johnson JL, et al: Cervical spine fracture patterns predictive of blunt vertebral artery injury. J Trauma 55:811-813, 2003

13. Cothren CC, Moore EE, Ray CE Jr, Ciesla DJ, Johnson JL, Moore JB, et al: Screening for blunt cerebrovascular injuries is cost-effective. Am J Surg 190:845-849, 2005

14. Delgado Almandoz JE, Schaefer PW, Kelly HR, Lev MH, Gonzalez RG, Romero JM: Multidetector CT angiography in the evaluation of acute blunt head and neck trauma: a proposed acute craniocervical trauma scoring system. Radiology 254:236-244, 2010

15. Desai NK, Kang J, Chokshi FH, Screening CT: Screening CT angiography for pediatric blunt cerebrovascular injury with emphasis on the cervical "seatbelt sign". AJNR Am J Neuroradiol 35:1836-1840, 2014

16. DiCocco JM, Fabian TC, Emmett KP, Magnotti LJ, Zarzaur BL, Khan N, et al: Functional outcomes following blunt cerebrovascular injury. J Trauma Acute Care Surg 74:955-960, 2013

17. Eastman AL, Chason DP, Perez CL, McAnulty AL, Minei JP: Computed tomographic angiography for the diagnosis of blunt cervical vascular injury: is it ready for primetime? J Trauma 60:925-929, 2006

18. Eastman AL, Muraliraj V, Sperry JL, Minei JP: CTA-based screening reduces time to diagnosis and stroke rate in blunt cervical vascular injury. J Trauma 67:551-556, 2009

19. Emmett KP, Fabian TC, DiCocco JM, Zarzaur BL, Croce MA: Improving the screening criteria for blunt cerebrovascular injury: the appropriate role for computed tomography angiography. J Trauma 70:1058-1065, 2011

20. Fassett DR, Dailey AT, Vaccaro AR: Vertebral artery injuries associated with cervical spine injuries: a review of the literature. J Spinal Disord Tech 21:252-258, 2008

21. Fleck SK, Langner S, Baldauf J, Kirsch M, Kohlmann T, Schroeder HW: Incidence of blunt craniocervical artery injuries: use of whole-body computed tomography trauma imaging with adapted computed tomography angiography. Neurosurgery 69:615-624, 2011

22. Friedman D, Flanders A, Thomas C, Millar W: Vertebral artery injury after acute cervical spine trauma: rate of occurrence as detected by MR angiography and assessment of clinical consequences. AJR Am J Roentgenol 164:443-449, 1995

23. Harrigan MR, Falola MI, Shannon CN, Westrick AC, Walters $\mathrm{BC}$ : Incidence and trends in the diagnosis of traumatic extracranial cerebrovascular injury in the nationwide inpatient sample database, 2003-2010. J Neurotrauma 31:1056-1062, 2014

24. Karamchandani R, Rajajee V, Pandey A: The role of neuroimaging in the latent period of blunt traumatic cerebrovascular injury. Open Neuroimaging J 5:225-231, 2011

25. Kaye D, Brasel KJ, Neideen T, Weigelt JA: Screening for blunt cerebrovascular injuries is cost-effective. J Trauma 70:1051-1057, 2011

26. Kerwin AJ, Bynoe RP, Murray J, Hudson ER, Close TP, Gifford RR, et al: Liberalized screening for blunt carotid and vertebral artery injuries is justified. J Trauma 51:308-314, 2001

27. Kopelman TR, Berardoni NE, O’Neill PJ, Hedayati P, Vail SJ, Pieri PG, et al: Risk factors for blunt cerebrovascular injury in children: do they mimic those seen in adults? J Trauma 71:559-564, 2011
28. Kopelman TR, Leeds S, Berardoni NE, O'Neill PJ, Hedayati $\mathrm{P}$, Vail SJ, et al: Incidence of blunt cerebrovascular injury in low-risk cervical spine fractures. Am J Surg 202:684-689, 2011

29. Kopriva BM, Smith RS, Yates CL, Helmer SD: Vertebral artery injury after blunt trauma without concomitant cervical or neurologic injuries: a screening conundrum. Am Surg 77:E72-E73, 2011

30. Kral T, Schaller C, Urbach H, Schramm J: Vertebral artery injury after cervical spine trauma: a prospective study. Zentralbl Neurochir 63:153-158, 2002

31. Löhrer L, Vieth V, Nassenstein I, Hartensuer R, Niederstadt $\mathrm{T}$, Raschke MJ, et al: Blunt cerebrovascular injuries in acute trauma care: a screening protocol. Eur Spine J 21:837-843, 2012

32. Miller PR, Fabian TC, Croce MA, Cagiannos C, Williams JS, Vang M, et al: Prospective screening for blunt cerebrovascular injuries: analysis of diagnostic modalities and outcomes. Ann Surg 236:386-395, 2002

33. Mitha AP, Kalb S, Ribas-Nijkerk JC, Solano J, McDougall CG, Albuquerque FC, et al: Clinical outcome after vertebral artery injury following blunt cervical spine trauma. World Neurosurg 80:399-404, 2013

34. Munera F, Foley M, Chokshi FH: Multi-detector row CT angiography of the neck in blunt trauma. Radiol Clin North Am 50:59-72, 2012

35. Parks NA, Croce MA: Use of computed tomography in the emergency room to evaluate blunt cerebrovascular injury. Adv Surg 46:205-217, 2012

36. Scott WW, Sharp S, Figueroa SA, Madden CJ, Rickert KL: Clinical and radiological outcomes following traumatic Grade 1 and 2 vertebral artery injuries: a 10-year retrospective analysis from a Level 1 trauma center. J Neurosurg 121:450-456, 2014

\section{Disclosures}

Dr. Steinmetz is a consultant for the following companies: Biomet Spine, DePuy Synthes, Globus Spine, Stryker, Intellirod. Dr. Mroz is owner of PearlDiver, Inc., and is a consultant for Stryker and Ceramtec.

\section{Author Contributions}

Conception and design: Smith, Lockwood, Tanenbaum, Lubelski, Seicean, Benzel, Mroz, Steinmetz. Acquisition of data: Smith, Lockwood, Lubelski, Benzel, Mroz, Steinmetz. Analysis and interpretation of data: Smith, Lockwood, Lubelski, Benzel, Mroz, Steinmetz. Drafting the article: Smith, Lockwood, Mroz, Steinmetz. Critically revising the article: Lockwood, Pace. Reviewed submitted version of manuscript: Pace.

\section{Supplemental Information}

\section{Previous Presentations}

Portions of this work were presented for an oral presentation at the Congress of Neurological Surgeons Meeting, Boston, Massachusetts, October 2014.

\section{Current Affiliation}

Dr. Lubelski: Department of Neurosurgery, Johns Hopkins Hospital, Baltimore, Maryland.

\section{Correspondence}

Gabriel A. Smith, Department of Neurological Surgery, Case Western Reserve University School of Medicine, 11100 Euclid Ave., 5th Fl. Hanna House, Cleveland, OH 44106. email: gabriel. smith@uhhospitals.org. 